\title{
A BALB/c murine lung alveolar carcinoma used to establish a surgical spontaneous metastasis model
}

\author{
Michael McLean ${ }^{1,2}$, Howard L. Wallace ${ }^{1, *}$, Atima Sharma ${ }^{1}$, Hank C. Hill ${ }^{3}$, Michael S. Sabel ${ }^{4}$ \& \\ Nejat K. Egilmez ${ }^{5}$ \\ ${ }^{1}$ Department of Microbiology and Immunology, and ${ }^{2}$ Department of Surgery, State University of New York at Buffalo, \\ Buffalo, New York, USA; ${ }^{3}$ Department of Surgery, Roswell Park Cancer Institute, Elm and Carlton Streets, Buffalo, New \\ York, USA; ${ }^{4}$ Division of Surgical Oncology, University of Michigan, Ann Arbor, Michigan, USA; ${ }^{5}$ J.G. Brown Cancer \\ Center and the Department of Microbiology and Immunology, University of Louisville, Louisville, Kentucky, USA
}

Received 15 February 2004; accepted in revised form 4 June 2004

Key words: cancer therapy, lung, murine, spontaneous metastasis, surgery, tumor model

\begin{abstract}
Line-1, a weakly immunogenic lung tumor cell line derived from the BALB/c mouse, metastasizes spontaneously to the lungs of mice following subcutaneous administration. The parameters that influence metastasis as well as the progression of metastatic lung disease following surgical resection of primary subcutaneous tumors were characterized. Histological analysis of the lungs obtained from mice bearing different size subcutaneous tumors demonstrated that $>90 \%$ of the mice developed micrometastatic disease in the lungs when the tumor exceeded $650 \mathrm{~mm}^{3}$ in size. Surgical resection of subcutaneous tumors resulted in the cure of primary disease in $95 \%$ of the mice. Macroscopic tumor nodules were grossly visible in the lungs of $75 \%$ of the mice 5 weeks after surgery. Serum amyloid A level correlated with primary tumor burden and was diagnostic for the presence of metastatic disease. The efficiency of metastasis, post-surgical primary tumor recurrence and long-term survival were significantly different between BALB/c mice obtained from different suppliers. The Line-1$\mathrm{BALB} / \mathrm{c}$ surgical metastasis model provides a clinically relevant tool for the evaluation of anti-cancer therapies, especially those that are designed to target long-term suppression of minimal residual disease following surgical intervention.
\end{abstract}

\section{Introduction}

Whereas studies with syngeneic transplantable murine tumor models have contributed greatly to our understanding of tumor biology and therapy, the majority of such studies have been performed in mice with localized disease. In the clinical setting however, most primary tumors can be cured by surgery but patients eventually succumb to metastatic disease. Therefore, the development of clinically relevant metastatic tumor models is critical to the identification of treatment protocols that are effective in patients. Since most tumor cell lines do not spontaneously metastasize, metastasis is generally induced by intravenous injection of tumor cells in transplantable murine tumor models. However, the relevance of intravenous metastasis models to spontaneously metastasizing disease is yet to be determined. Several transplantable tumor cell lines that spontaneously metastasize from a subcutaneous site to different organs have been described [1-4]. With few exceptions [4], these models have

\footnotetext{
*This paper is dedicated to the memory of Dr. Howard L. Wallace.

Correspondence to: Nejat Egilmez, Assistant Professor, J.G. Brown Cancer Center, University of Louisville, 580 S. Preston Street, Room 119C, Louisville, KY 40202, USA. Tel: +1-502-852-4030; Fax: +1-502-852-2123 E-mail: nejat.egilmez@louisville.edu
}

not been characterized in detail and their use has been limited.

Line-1 is a weakly immunogenic tumor cell line that was derived from a spontaneously arising lung alveolar carcinoma of the BALB/c mouse [2]. When injected subcutaneously, Line-1 cells grow rapidly and metastasize spontaneously to the lungs [2]. The metastatic nature of this cell line is associated with low Class I MHC expression, which is inducible by interferon-gamma (IFN $\gamma$ ) or dimethyl sulfoxide (DMSO) [5]. Line-1-BALB/c model has been utilized to investigate the role of cytotoxic T-cells and natural killer (NK) cells as well as the involvement of the transcription factor nuclear factor kappa $\mathrm{B}(\mathrm{NF}-\kappa \mathrm{B})$ in tumor metastasis [6, 7]. Several cancer immunotherapy protocols including cytokine-based therapies [8,9], gene-therapy based approaches involving co-stimulatory molecules [10] and suicide genes [11] have also been evaluated in this system.

While the metastatic properties of Line-1 cells are well established, the Line-1-BALB/c spontaneous metastasis model has not been utilized to evaluate strategies targeting disseminated disease. To this end, we recently developed a surgical embodiment of this model to test the anti-tumor efficacy of a tumor immunotherapy protocol [12, 13]. In this model, primary subcutaneous Line-1 tumors are induced and 
are allowed to metastasize to the lungs of mice. Treatment is then administered either prior to or after surgical debulking of the primary tumor, and primary tumor recurrence as well as the progression of metastatic disease is monitored. Here we describe this clinically relevant model which was designed for the evaluation of adjuvant therapies for the treatment of disseminated disease.

\section{Methods}

\section{Mice and cell lines}

Female BALB/c mice at six to eight weeks of age were obtained either from Jackson Laboratories (Bar Harbor, Maine) or from Taconic Laboratories (Germantown, New York). Mice were maintained five per cage under standard housing conditions. Animals were used in the studies at 8-12 weeks of age. Line-1 (a BALB/c lung alveolar carcinoma cell line) was a gift from Dr John G. Frelinger (University of Rochester, School of Medicine and Dentistry, Rochester, New York).

\section{Tumor cell injections}

Mice were injected subcutaneously behind the neck just above the scapula with $1 \times 10^{7}$ tumor cells (in $200 \mu$ l saline). Tumor growth was monitored by measurement of tumor diameter with engineer's calipers twice a week. Tumor volume was determined by the formula $a \times b^{2} / 2$ where $a$ and $b$ are the longest and the shortest perpendicular diameters of the tumor, respectively. When the tumors reached the desired size $\left(250-1500 \mathrm{~mm}^{3}\right)$ the mice underwent surgery or were sacrificed and evaluated for metastasis. Only mice with circular/oblong tumors were included in the studies. Animals with irregular tumors were not used due to imprecise tumor volume determination.

\section{Surgery}

All surgical procedures were performed in a laminar flow surgical hood under aseptic conditions. Surgical instruments were sterilized by autoclaving and personnel wore disposable sterile gown, mask, head cover and gloves. Mice were injected intra-peritoneally with Avertin $(0.012 \mathrm{ml} / \mathrm{g}$ weight $)$ to achieve a surgical phase of anesthesia and were placed on a sterile surgical diaper over a heated circulating water mat to maintain body warmth. The skin around the subcutaneous tumor was shaved, scrubbed with antiseptic soap, prepared with alcohol and painted with betadine. A skin incision was made over the circumferential length of the tumor with micro-dissection scissors and tumor tissue was isolated by sharp dissection. Hemostasis was established by gentle pressure or electrocautery of the major vessels entering into the tumor tissue. The isolated tumor tissue was removed and the skin was closed with simple interrupted sutures using monofilament 6-0 nylon or 6-0 vicryl. Mice were injected i.p. with $0.2 \mathrm{ml}$ of sterile saline, kept warm and observed until they recovered from anesthesia. When fully recovered they were returned to their respective cages. The entire surgery lasted 10 to 15 min per mouse. Marcaine (50\% solution) was topically applied to the wound 3 times a day for 2 days to reduce post-surgical discomfort.

\section{Analysis of micrometastatic disease}

Mice bearing different size tumors (or at different time points after surgery) were sacrificed by $\mathrm{CO}_{2}$ inhalation, the lungs were removed, rinsed with saline and fixed in $10 \%$ formalin. Formalin-fixed lungs were embedded in paraffin and serial sections (5 $\mu \mathrm{m}$ thick) were cut throughout the entire lung tissue. Every 25 th section was saved and stained with hematoxylin and eosin (H \& E) using standard procedures. H \& E stained lung sections were analyzed for tumor microscopically under $40 \times$ and $100 \times$ magnification. Tumor nodules were identified as densely packed large mitotic cells staining strongly with eosin against the normal lung tissue background.

\section{Analysis of macrometastatic disease}

Mice bearing different size tumors (or at different time points after surgery) were sacrificed by $\mathrm{CO}_{2}$ inhalation, the lungs were removed and were stained with India ink as previously described [14]. White tumor nodules against a black lung background were counted under a dissecting microscope. Tumor area was determined using the formula $\pi \mathrm{ab} / 2$, where $\mathrm{a}$ and $\mathrm{b}$ are the shortest and longest perpendicular dimensions of the tumor nodule.

\section{Quantification of serum TNF $\alpha, I L-1 \beta$ and $S A A$}

Serum $\mathrm{TNF} \alpha$, IL- $1 \beta$ and amyloid A levels were determined using the murine TNF $\alpha$ (Endogen, Rockford, Illinois), IL-1 $\beta$ (Endogen) and SAA-specific (Biosource, Camarillo, California) ELISA kits as recommended by the manufacturer.

\section{Long-term survival studies}

Mice were injected with tumor cells and primary tumors were allowed to reach an average size of $1000 \mathrm{~mm}^{3}$. They were then surgically resected as described above and mice were monitored for primary tumor recurrence and signs of systemic disease. Animals were sacrificed at the first sign of dyspnea, paralysis or cachexia.

\section{Statistical methods}

Student's $t$-test (unpaired) was utilized to determine the significance of the differences between groups in macrometastasis studies as well as in SAA analyses. Linear regression analysis was used to determine the correlation coefficients for tumor volume vs weight, and SAA concentration vs primary tumor volume (Stata SE/8.2, Stata Corp., College Station, Texas). Receiver-operator characteristic analysis was employed to determine the significance of selected SAA threshold concentrations as diagnostic for micrometastatic disease (Stata SE/8.2, Stata Corp., College Station, Texas). 


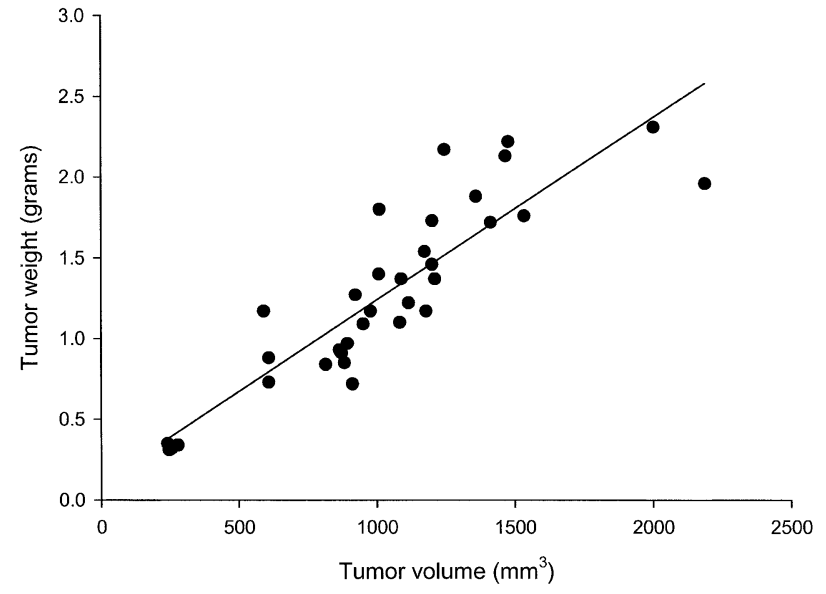

Figure 1. Calculated primary tumor volume versus actual weight. Subcutaneous tumors were induced and allowed to reach different sizes. Tumor volume was determined for each mouse as described in the methods. The mice were sacrificed, tumors were removed and weighed. Tumor volume was then plotted against actual tumor weight for each individual $(n=35)$. Linear regression analysis demonstrated that the correlation between tumor volume and weight was highly significant $(r=0.793, P=0.0001)$.

Table 1. Lung micrometastasis in the Line-1-BALB/c spontaneous tumor metastasis model.

\begin{tabular}{ccccc}
\hline \multicolumn{2}{c}{ Primary tumor } & & \multicolumn{2}{c}{ Micrometastasis } \\
\cline { 1 - 2 } \cline { 5 - 5 } Size $\left(\mathrm{mm}^{3}\right)$ & Days & & $\%$ of mice & $\%$ of sections \\
\hline $259 \pm 15$ & $10.8 \pm 1.8$ & & $80(4 / 5)$ & $34(10 / 29)$ \\
$642 \pm 86$ & $14.6 \pm 3.6$ & & $100(5 / 5)$ & $62(21 / 34)$ \\
$1105 \pm 180$ & $16.6 \pm 2.7$ & & $100(4 / 4)$ & $72(18 / 25)$ \\
\hline
\end{tabular}

The significance of the differences between survival patterns was determined by log-rank analysis (GraphPad Prism, GraphPad Software, Inc., San Diego, California).

\section{Results}

\section{Characterization of lung metastasis}

We initially attempted to establish the minimum primary tumor size and/or growth period required to achieve consistent metastasis to the lungs. In most studies, subcutaneous tumor growth is monitored by measurement of tumor diameter. However, measurement of tumor diameter is not always straightforward and the formulas that are used to determine volume based on these measurements may not necessarily be accurate. To determine whether the formula we used reflected the tumor size accurately, we compared the calculated tumor volume to tumor weight. Subcutaneous tumors were induced, allowed to reach different sizes, measured and then surgically resected and weighed. The calculated tumor volume was then plotted against weight. The results are shown in Figure 1. These data establish that our formula for estimating tumor volume reflected the actual tumor size accurately and thus could be used to determine the relationship between primary tumor size and efficiency of metastasis.
The relationship between primary tumor size and metastasis kinetics was investigated by histological analysis of the lungs in mice bearing primary tumors of different sizes. Mice were injected with Line-1 cells subcutaneously and tumors were monitored until they reached an average of 250 , 650 and $1100 \mathrm{~mm}^{3}$ in size. The mice in each group were then sacrificed, the lungs were removed and histological sections were prepared from each lung. Histological sections obtained throughout the lung were analyzed for evidence of metastatic tumor nodules by microscopic examination of each section. The results are summarized in Table 1 . While few small lesions were observed in mice bearing small $\left(250 \mathrm{~mm}^{3}\right)$ primary tumors, most sections were found to be tumor-free (Table 1). In mice bearing primary tumors that were $650 \mathrm{~mm}^{3}$ on average, however, metastatic nodules were more easily distinguishable and numerous. In fact lungs from all mice in this group were positive for metastasis and the majority of the sections (62\%) showed evidence of tumor. In mice bearing larger tumors, metastatic nodules were not larger in size but the percentage of lung sections that were positive for metastasis was slightly higher $(72 \%$, Table 1). The average size of primary tumors correlated well with time of tumor growth in this study (Table 1). These results establish that while evidence of micrometastatic disease can be found in the lungs of mice bearing primary tumors as small as $250 \mathrm{~mm}^{3}$, development of multi-focal, wellestablished disease is achieved only when the primary tumor exceeds $650 \mathrm{~mm}^{3}$ in size.

The primary goal of this study was to develop a model that could be utilized to evaluate adjuvant anti-cancer strategies in combination with surgery. To this end, the progression of metastatic disease after surgical removal of the primary tumor was evaluated. Subcutaneous tumors were induced and were allowed to reach $\sim 1000 \mathrm{~mm}^{3}$ in size to ensure efficient metastasis to the lungs. Primary tumors were then resected and the post-surgical progression of metastatic lung tumors was monitored by histological analysis of lungs 7, 14 and 21 days after surgery. Representative lung sections from mice with efficient metastasis and the results of microscopic analyses of the lungs are shown in Figure 2 and Table 2, respectively. While the percentage of tumor-positive lung sections remained unchanged after surgery (Table 2), metastatic tumors grew rapidly and normal lung tissue was progressively replaced by tumor in individuals with established micrometastasis (Figure 2). Approximately $15 \%(2 / 14)$ mice were found to be free of lung tumors histologically in this study indicating that metastasis is not $100 \%$ efficient even when the primary tumor is as large as $1000 \mathrm{~mm}^{3}$ (Table 2). Overall, combined results from Tables 1 and 2 show that when the primary tumor exceeds $650 \mathrm{~mm}^{3}$ in size, $>90 \%(21 / 23)$ of the mice develop micrometastatic lung disease.

Although the histological characterization of microscopic disease provided valuable data on the kinetics of metastasis and the post-surgical growth pattern of lung tumors, microscopic analysis of metastatic lung tumors as a way of monitoring disease progression is labor intensive and expensive. A simpler approach, which is used in 

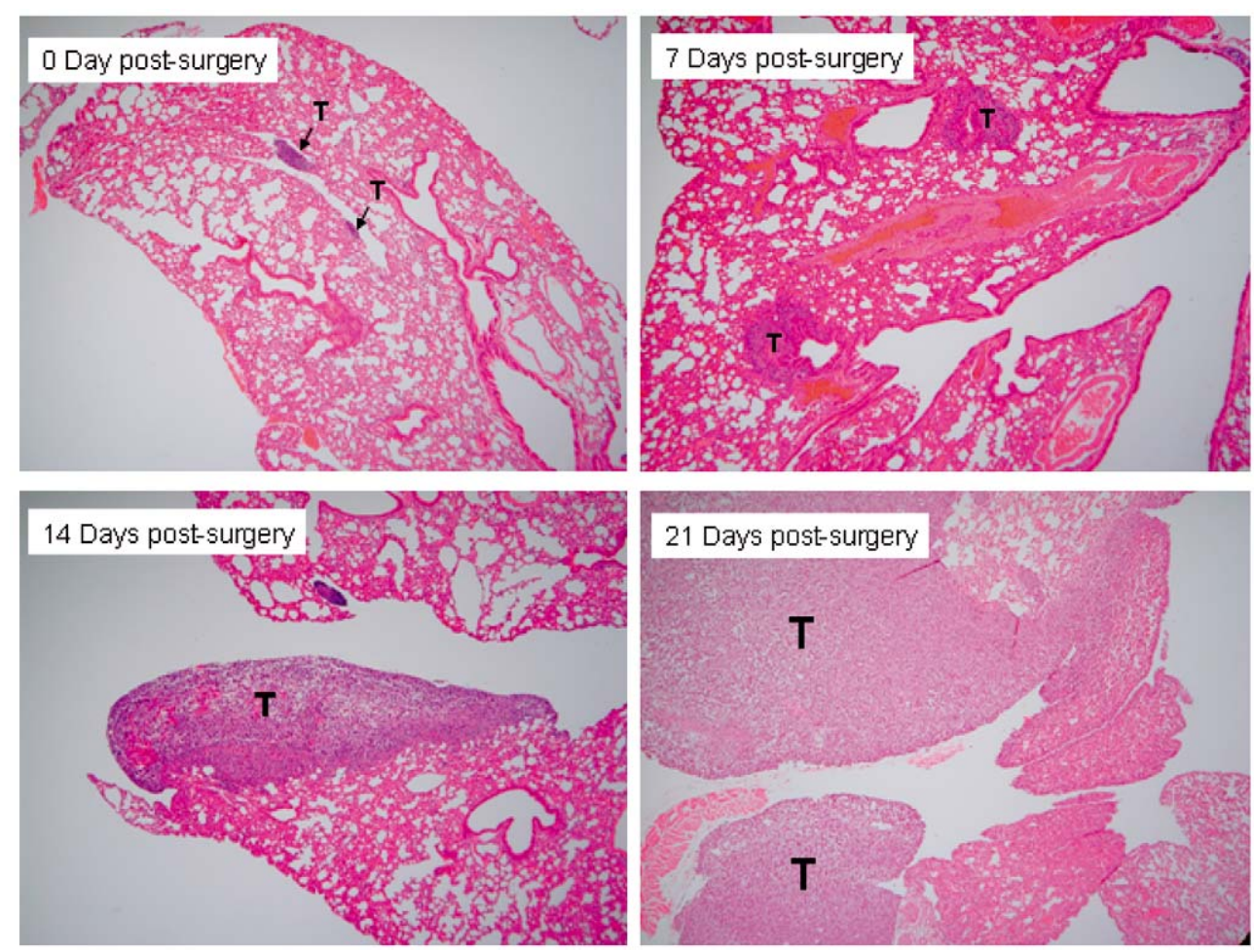

Figure 2. Post-surgical growth of lung micrometastases. Primary tumors were allowed to grow to a size of $1075 \pm 199 \mathrm{~mm}^{3}$ and were surgically removed. Mice were sacrificed either on the day of surgery, or 7, 14 and 21 days after surgery, and the lungs were analyzed microscopically for tumor burden as described in the methods. The micrographs shown are representative for each time point. ' $\mathrm{T}$ ' indicates tumor tissue $(n=5$, magnification $=40 \times)$.

Table 2. Post-operative Lung micrometastasis in the Line-1-BALB/c spontaneous tumor metastasis model.

\begin{tabular}{ccl}
\hline Post-operative day $^{\mathrm{a}}$ & $\%$ of mice positive & $\%$ of sections positive \\
\hline 7 & $75(3 / 4)$ & $58(30 / 52)$ \\
14 & $100(5 / 5)$ & $69(33 / 48)$ \\
21 & $80(4 / 5)$ & $63(38 / 60)$ \\
\hline
\end{tabular}

${ }^{\mathrm{a} A v e r a g e ~ p r i m a r y ~ t u m o r ~ s i z e ~ a t ~ t i m e ~ o f ~ s u r g e r y ~ w a s ~} 1075 \pm 199 \mathrm{~mm}^{3}$.

the majority of published studies, involves the analysis of the number and/or size of macroscopic lung tumors. In the studies described above (Table 1), macroscopic tumor nodules were observed in some of the mice bearing primary tumors $\geq 650 \mathrm{~mm}^{3}$ in size. Moreover, post-surgical analysis of lung sections demonstrated that metastatic lung tumors progressed rapidly after the resection of the primary tumor (Figure 2) and that tumor nodules were macroscopically visible by three weeks after surgery in the lungs of most animals. To determine whether the macroscopic lung tumor nodule numbers could be used as a quantitative marker for post-surgical disease progression, we analyzed the lungs of mice at 1,3 and 5 weeks after surgical removal of the primary tumor $\left(890 \pm 240 \mathrm{~mm}^{3}\right)$. At week 1 mice had an average of $0.7 \pm 1.1$ macroscopic lung tumors $(n=10)$. At weeks 3 and 5, the average number of lung tumors were $3.8 \pm 3.5$ and $3.5 \pm 3.8$ nodules per lung, respectively $(n=8$ for both groups). The differences between week 1 and weeks 3 or 5 were statistically significant $(P<0.04)$. There was no difference between weeks 3 and $5(P=0.89)$. Thus, short- term tumor growth could be monitored by macroscopic analysis of the lungs between weeks 3-5 post-surgery. In these studies variation in both tumor number [1-10] and size $(0.2-4 \mathrm{~mm})$ was observed between individual mice in several independent studies (data not shown). The large standard deviations within each group were in part due to this variation as well as the presence of several mice that were free of macroscopic disease $(25 \%, 4 / 16)$.

\section{Serum amyloid $A$ as an indirect tumor marker}

The studies described above demonstrate that quantification of macroscopic lung tumor burden can provide a simple method for evaluating the efficacy of a given treatment in the Line-1-BALB/c model. On the other hand, this approach requires that the mice are sacrificed at a selected time-point. The identification of a soluble tumor marker that can be detected in the serum would provide the ability to monitor tumor burden continuously without sacrificing the mice. In addition, such a marker can be used as a diagnostic tool to identify mice with poor vs efficient metastasis prior to treatment thus possibly reducing the variability caused by the differences in pre-treatment tumor burden among individual mice. Serum protein biomarkers have been identified for several human cancers [15]. However, similar endogenous tumor markers are not currently available for murine tumor models.

Human serum amyloid A (SAA), an acute phase liver protein which is produced rapidly in response to inflammatory stimuli, has been shown to correlate with disease stage and prognosis in cancer patients [16-18]. The correlation 

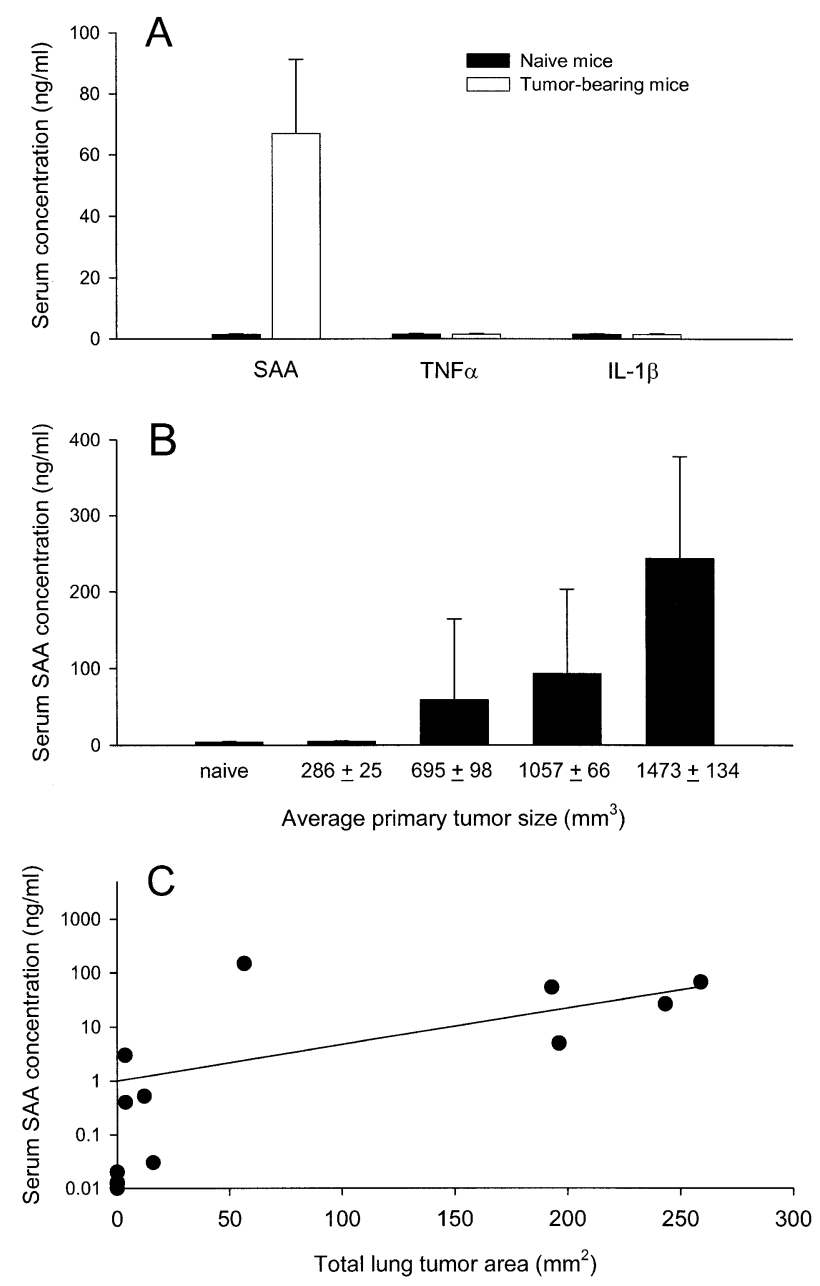

Figure 3. SAA level versus tumor burden. A. Mice with large subcutaneous tumors $\left(1581 \pm 248 \mathrm{~mm}^{3}\right)$ or that were tumor-free were bled and serum SAA, TNF $\alpha$ and IL- $1 \beta$ levels were determined. Bars $=$ standard deviation, $n=3$ per group. B. Primary tumors were induced and allowed to grow to selected sizes $(n=6,11,10,11$ and 7 for naïve mice, and mice with average tumor sizes of $286 \mathrm{~mm}^{3}, 695 \mathrm{~mm}^{3}, 1057 \mathrm{~mm}^{3}$, $1473 \mathrm{~mm}^{3}$, respectively). SAA levels were determined for each individual and the average SAA concentration for each size group was plotted against the corresponding tumor group. Linear regression analysis demonstrated a significant correlation between tumor size and SAA level $(r=0.523$ with a $P$-value of 0.0006$)$. C. Subcutaneous tumors were induced, allowed to grow to different sizes $\left(555-1879 \mathrm{~mm}^{3}\right)$ and were removed surgically. Serum was collected from each individual 5 weeks after surgery and the mice were sacrificed. Macroscopically visible total lung tumor area was determined for each individual and plotted against SAA values for each individual. Linear regression analysis did not show a significant correlation between metastatic tumor area and SAA level $(r=0.406$ with a $P$-value of 0.133). Receiver-operator characteristic (ROC) analysis demonstrated a significant predictive threshold for an SAA concentration of $\geq 0.05 \mathrm{ng} / \mathrm{ml}$ and the presence of metastatic lung disease (ROC area of 0.818 with a $95 \%$ confidence interval between 0.58 and 1.0).

between SAA and tumor burden has not been investigated in murine tumor models. To determine whether SAA levels correlated with tumor presence in the Line-1-BALB/c model, serum SAA levels in tumor-bearing and naïve mice were measured. The changes in the serum levels of the inflammatory cytokines IL $1 \beta$ and $\mathrm{TNF} \alpha$, which have been shown to induce SAA expression in response to inflammatory stimuli [19], were also determined. The results shown in Figure 3A demonstrate that tumor-bearing mice have elev- ated levels of SAA in their sera in comparison to naïve mice. On the other hand, no increases in IL- $1 \beta$ or $\mathrm{TNF} \alpha$ could be detected in the sera of mice with established tumors as compared to tumor-free naïve mice.

To determine whether the serum SAA levels correlated with tumor burden in our model, we measured both primary tumor size and serum SAA levels in individuals with different size subcutaneous tumors. The average SAA level was then plotted against average tumor size for each size group. The results are shown in Figure 3B. These results demonstrate that average serum SAA levels increased with increasing tumor size. While the SAA levels within each group displayed considerable variability (Figure 3B), the correlation between primary tumor size and SAA level was significant (Figure 3, legend).

Finally we examined whether the post-surgical SAA levels correlated with metastatic tumor burden in the lungs. Primary tumors were allowed to grow to an average size of $1000 \mathrm{~mm}^{3}$ and then were surgically resected. The SAA levels remained high within the first 3-4 days after surgery due possibly to surgical trauma but then decreased to near background levels by day 7 (data not shown). Five weeks after surgery, blood was collected, the mice were sacrificed and lung tumor burden was quantified. Lung tumor burden (total tumor surface area) was then plotted against serum SAA level for each mouse. The results are shown in Figure $3 \mathrm{C}$. Whereas the correlation between lung tumor burden and SAA level was not statistically significant (Figure 3, legend), an SAA level of $>0.05 \mathrm{ng} / \mathrm{ml}$ was predictive for the presence of metastatic lung tumors (Figure 3, legend). Thus SAA is more useful as a diagnostic (tumor vs no tumor) than a quantitative marker for metastatic disease in this model.

\section{Efficiency of metastasis in BALB/c mice obtained from different suppliers}

The above studies were performed with BALB/c mice that were obtained from Jackson laboratories. In separate studies we also tested BALB/c mice from Taconic laboratories and noticed that Line-1 tumors metastasized more aggressively in the Taconic BALB/c. To determine if this preliminary observation was valid, we decided to compare the pattern of metastasis in mice that were obtained from Jackson vs Taconic laboratories. Primary subcutaneous tumors were induced, allowed to grow to an average size of $750 \mathrm{~mm}^{3}$, and were then surgically resected. Mice were sacrificed five weeks after surgery and macroscopic lung tumor burden was quantified. BALB/c mice that were obtained from Jackson laboratories had an average of $1.6 \pm 3.4$ tumors/lung while the Taconic mice had $5.5 \pm 4.2$ tumors/lung $(n=8$ per group, $P=0.035$ ), confirming the preliminary observation that Line- 1 cells metastasized more efficiently in the Taconic $\mathrm{BALB} / \mathrm{c}$ mice.

All prior studies addressed the short-term kinetics (up to five weeks) of lung metastasis in the Line-1-BALB/c model. We next evaluated the post-surgical survival patterns for both Taconic and Jackson Laboratory mice to determine the long-term growth kinetics of metastatic disease. The results are shown in Figure 4. The data demonstrate that mice in 


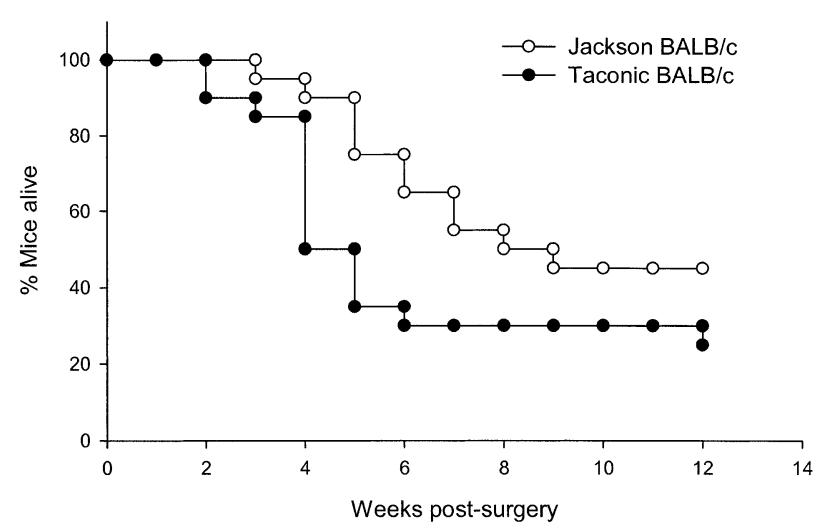

Figure 4. Post-surgical survival in BALB/c mice obtained from different sources. Primary tumors were induced and were surgically removed upon reaching an average size of $997 \pm 168 \mathrm{~mm}^{3}$ for Jackson and $1011 \pm 188 \mathrm{~mm}^{3}$ for Taconic mice. Mice were then monitored for long-term survival $(n=20$ per group). The difference between the two groups was significant at 6 weeks, but not at 12 weeks as determined by log-rank analysis $(P=0.012$ and 0.055 , respectively).

each group began dying of metastatic tumors within three to four weeks of surgery. Death from metastatic disease occurred more rapidly in the Taconic mice, with a median survival of 4.5 weeks as compared to 8 weeks for the Jackson mice. By week 12 post-surgery, $75 \%$ of the Taconic mice had died from metastatic disease as compared to $55 \%$ of the Jackson mice. Primary tumor recurrence during the 12week post-surgical period was also significantly higher in the Taconic BALB/c $(30 \%, 6 / 20)$ as compared to Jackson BALB/c (5\%, 1/20). These results confirm that Line-1 cells metastasize more aggressively in the BALB/c mice obtained from Taconic Laboratories as compared to those purchased from Jackson Laboratories. The data also demonstrate that metastatic disease, once established, progresses rapidly, and that most mice die from lung tumors within eight weeks of surgery.

\section{Discussion}

The studies described here establish that the Line-1-BALB/c spontaneous metastasis model provides a reliable system for the evaluation of anti-cancer strategies, especially those that target post-surgical micrometastatic disease. The availability of such a model is particularly relevant to immunotherapeutic strategies, an approach which is now recognized as most effective in the minimal residual disease setting [20]. It is expected that the information provided here will expand the use of this model among cancer investigators.

Whereas the above studies demonstrated the utility of the Line-1-BALB/c surgical metastasis model, they also identified some of its limitations. For example, although the great majority $(90 \%)$ of the mice developed micrometastases in the lung once the average size of the primary tumor exceeded $650 \mathrm{~mm}^{3}$, micrometastatic lesions progressed to macroscopic disease in $75 \%$ of these mice. This is not surprising as studies by others have demonstrated that only a subset of micrometastases persist to form vascularized macrometastatic nodules [21]. In addition, we found that metastasis was less efficient and variability was more pronounced in the Jackson Laboratory mice as compared to the Taconic Laboratory mice. Both the short-term development pattern of lung tumors and long-term post-surgical survival of mice (Figure 4) demonstrate that BALB/c mice obtained from Taconic laboratories are more susceptible to Line-1 metastasis. It is well established that different strains of mice react dissimilarly to immune challenge due to genetic variability [22]. On the other hand, such differences would not be expected, or should be minimal, within the same inbred strain. One possible explanation for the observed differences between the Jackson and the Taconic BALB/c is genetic drift. Intra-strain genetic drift can occur if populations remain segregated for extended periods [23]. Our data support the notion that genetic drift between the BALB/c colonies of Jackson and Taconic laboratories has reached a level where it can impact physiological responses differentially, resulting in significant experimental variability. The higher rate of metastasis, lower variability and more aggressive postsurgical metastatic disease progression in Taconic BALB/c suggests that this substrain should be preferred in future studies.

A potentially valuable finding in this study was the demonstration that serum SAA levels increase in tumorbearing mice. Analysis of SAA levels in mice with increasing primary tumor burden showed that SAA correlated with tumor burden and could be used as a marker for monitoring tumor progression (Figures 3A and B). However, SAA level in post-surgical mice with macroscopic lung tumors did not correlate with metastatic tumor burden quantitatively, but instead was indicative of tumor absence or presence. The relatively low metastatic tumor burden in post-surgical mice may not induce sufficient inflammatory activity thus making it difficult to use SAA as a quantitative marker in this setting. This notion is supported by the very low/background levels of SAA found in mice bearing small primary tumors $\left(\leq 250 \mathrm{~mm}^{3}\right.$, Figure $\left.3 \mathrm{~B}\right)$. On the other hand, the results shown in Figure 3C demonstrate that SAA can still be useful as a diagnostic marker for metastatic disease in post-surgical mice. Whether this finding applies to other murine tumor models remains to be determined.

\section{Acknowledgements}

The authors would like to thank Dr. Richard B. Bankert for generously making his laboratory facilities available during these studies. This work was supported in part by NIH/NCI grant CA085097-01A1 to N.K.E.

\section{References}

1. Markovic SM and Murasko DM. Neoadjuvant immunotherapy with interferon of the spontaneously metastasizing murine B16F10L melanoma. Int J Cancer 1990; 45: 788-94.

2. Yuhas MJ and Pazmino NH. Inhibition of subcutaneously growing Line 1 carcinomas due to metastatic spread. Cancer Res 1974; 34 : 2005-10. 
3. Cavallo F, Di Carlo E, Butera M et al. Immune events associated with the cure of established tumors and spontaneous metastases by local and systemic interleukin-12. Cancer Res 1999: 59: 414-21.

4. Pulaski BA, Terman DS, Khan S et al. Cooperativity of Staphylococcal aureus enterotoxin $\mathrm{B}$ superantigen, major histocompatibility complex class II, and CD 80 for immunotherapy of advanced spontaneous metastases in a clinically relevant postoperative mouse breast cancer model. Cancer Res 2000; 60: 2710-5.

5. Bahler DW, Lord EM. Enhanced immune recognition of H-2 antigendeficient murine lung carcinoma cells following treatment with dimethylsulfoxide. Cancer Res 1985; 45(12):6362-5.

6. Cerosaletti KM, Blieden TM, Harwell LW et al. Alteration of the metastatic potential of Line-1 lung carcinoma cells: opposite effects of Class I antigen induction by interferons versus DMSO or gene transfection. Cell Immunol 1990; 127: 299-310.

7. Andela VB, Schwarz EM, Puzas JE et al. Tumor metastasis and the reciprocal regulation of prometastatic and antimetastatic factors by nuclear factor kappaB. Cancer Res 2000; 60(23): 6557-62.

8. McAdam AJ, Pulaski BA, Harkins SS et al. Synergistic effects of coexpression of the TH1 cytokines IL-2 and IFN-gamma on generation of murine tumor-reactive cytotoxic cells. Int J Cancer 1995; 61(5): 628-34.

9. McAdam AJ, Pulaski BA, Storozynsky E et al. Analysis of the effect of cytokines (interleukins 2, 3, 4, and 6, granulocyte-monocyte colony-stimulating factor, and interferon-gamma) on generation of primary cytotoxic $\mathrm{T}$ lymphocytes against a weakly immunogenic tumor. Cell Immunol 1995; 165(2): 183-92.

10. Yeh KY, Pulaski BA, Woods ML et al. B7-1 enhances natural killer cell-mediated cytotoxicity and inhibits tumor growth of a poorly immunogenic murine carcinoma. Cell Immunol 1995; 165(2): 217-24.

11. Miller PW, Sharma S, Stolina, M, et al. Dendritic cells augment granulocyte-macrophage colony-stimulating factor (GM-CSF)/herpes simplex virus thymidine kinase-mediated gene therapy of cancer. Cancer Gene Ther 1998; 5(6): 380-9.

12. Sabel MS, Hill HC, Yong YS et al. Neoadjuvant therapy with IL-12 loaded microspheres reduces local recurrence and distant metastases. Surgery 2001; 130(3): 470-8.
13. Hill HC, Conway TF, Sabel MS et al. Cancer immunotherapy with interleukin-12 and granulocyte-macrophage colony-stimulating factor-encapsulated microspheres: Coinduction of innate and adaptive immunity and cure of disseminated disease. Cancer Res 2002; 62: 7254-63.

14. Wexler H. Accurate identification of experimental pulmonary metastases. J Natl Cancer Inst 1966; 36: 641-5.

15. Johnson PJ. A framework for the molecular classification of circulating tumor markers. Ann N Y Acad Sci 2001; 945: 8-21.

16. Biran H, Friedman N, Neumann L et al. Serum amyloid A (SAA) variations in patients with cancer: correlation with disease activity, stage, primary site, and prognosis. J Clin Pathol 1986; 39: 794-7.

17. Kimura M, Tomita Y, Imai T et al. Significance of serum amyloid A on the prognosis in patients with renal carcinoma. Cancer 2001; 92(8): 2072-5.

18. Glojnaric I, Casi M-T, Simic D et al. Serum amyloid A protein (SAA) in colorectal carcinoma. Clin Chem Lab Med 2001; 39(2): 129-33.

19. Ghezzi P and Sipe JD. Dexamethasone modulation of LPS, IL-1 and TNF stimulated serum amyloid A synthesis in mice. Lymphokine Res 1988; 7(2): 157-66.

20. Bendandi M, Gocke CD, Kobrin $\mathrm{CB}$ et al. Complete molecular remissions induced by patient-specific vaccination plus granulocytemonocyte colony-stimulating factor against lymphoma. Nat. Med 1999; 5(10): 1171-7.

21. MacDonald IC, Groom AC, Chambers AF. Cancer spread and micrometastasis development: quantitative approaches for in vivo models. BioEssays 2002; 24: 885-93.

22. Barral-Netto M, Cardoso SA, Barral A. Different patterns of disease in two inbred mouse strains infected with a clone of Leishmania mexicana amazonensis. Acta Trop 1987; 44(1): 5-11.

23. Wotjak CT. C57Black/BOX? The importance of exact mouse strain nomenclature. Trends Genet 2003; 19(4): 183-4. 